\title{
Assessing the Level of Safety for Performance Based and Prescriptive Structural Fire Design of Steel Structures
}

\author{
GIANLUCA DE SANCTIS ${ }^{1}$, MICHAEL H. FABER ${ }^{2}$ and MARIO FONTANA ${ }^{1}$ \\ ${ }^{1}$ ETH Zurich \\ Institute of Structural Engineering \\ Zurich, Switzerland \\ ${ }^{2}$ Technical University of Denmark \\ Department of Civil Engineering \\ Lyngby, Denmark
}

\begin{abstract}
The level of safety in structural fire safety is implemented by combining passive and active fire safety measures. Prescriptive and some performance based codes provide requirements to achieve this level of safety without explicitly quantifying it. Here, a reliability based method is used to quantify the level of safety of a design. A generic representation of the building facilitates the application of the methodology on different buildings and to consider the requirements of the codes. Engineering models are used to consider the effect of fire safety measures including the fire brigade intervention under realistic fire conditions. The uncertainties associated with these engineering models are considered through a probabilistic approach. The reliability of the structure is assessed through an advanced Monte Carlo technique called subset simulation. The methodology is applied for retail buildings. The benefits using performance based codes are addressed and compared with the results of prescriptive codes. The methodology can be used for verifying equivalency in fire safety design as well.
\end{abstract}

KEYWORDS: reliability, performance based design, structural design, fire brigade intervention

\section{NOMENCLATURE}

\section{Variables}

$X \quad$ Random variable

$x \quad$ Realization of $X$

$\mathbf{X}$ Vector of random variables

$\mathbf{x} \quad$ Vector of realizations of $\mathbf{X}$

\section{Subscripts}

d Design value

$k \quad$ Characteristic value

fi Accidental fire design

opt Optimum

\author{
Operators \\ $f_{X}(x) \quad$ Probability density function of $X$ \\ $P(A) \quad$ Probability of an event $A$ \\ $P(A \mid B) \quad$ Conditional probability of $A$ given $B$ \\ $E[X] \quad$ Expected value / mean value of $X$ \\ $\operatorname{CoV}[X] \quad$ Coefficient of variation of $X$ \\ $\theta(t) \quad$ Steel temperature \\ $\Theta(t) \quad$ Gas temperature
}

\section{INTRODUCTION}

Codes describe how fire safety can be achieved. Their requirements are usually set by the fire authorities. By comparing different countries it is shown that fire safety requirements for buildings differ, even for equal risk classes (Schleich et al. [1]). The application of performance based codes is questioned in many countries by the fire authorities. One reason to question the performance based codes is the lack of proof for equivalency to the prescriptive design given in traditional regulation.

In this paper a reliability based method is used to quantify the level of safety which is achieved by implementing either a prescriptive design or a performance based design. This method provides a common quantification of the level of safety. Thus, different designs can be compared and the equivalency can be assessed. The outline of the paper is illustrated in Fig. 1.

First, it is discussed how the level of safety in structural design is achieved using a prescriptive or a performance based design. Then, it is explained how the level of safety is quantified through a reliability based method. This quantification is in agreement with JCSS [2] and with EN 1990 [3]. Structural 
reliability assessment in fire has already been done e.g. by Schleich et al. [1] and Hosser et al. [4]. They use a simplified calculation method to assess the reliability of structure as basis for code calibration.

In this paper the building is represented generically by a selection of building properties. This facilitates parametric studies of the effect of different building properties on the safety level. The generic design fulfills the state of the art of structural design and implement the requirements of the codes, e.g. persistent and accidental (fire) design situation. Based on this generic design the safety level of a structure is achieved by providing a certain amount of required mechanical resistance and a certain amount of required thermal resistance.

To assess the level of safety the behavior of a well-designed structure under realistic fire conditions is studied. Because real conditions are always associated with uncertainties, due to natural variability and lack of knowledge, a probabilistic approach is used to consider those uncertainties. Engineering models are introduced to consider the physical interaction of the model parameters and allow assessing the effect of passive and active fire safety measures quantitatively.

The annual probability of failure, which is used as a measure for the level of safety, is achieved by a reliability analysis. The reliability of the structure is assessed through an advanced Monte Carlo analysis called subset simulation introduced by Beck \& $\mathrm{Au}$ [6] and applied to fire engineering by Au et al. [7].

The reliability analysis is used, on one hand, to compare a performance based structural fire design according to EN 1991-1-2 [5] with a prescriptive design. On the other hand, the effect of the different treatment of buildings parameters by the codes on the reliability is assessed. This approach can be used to proof the equivalency of alternative design solutions with standard design solutions. In this paper, the equivalency of a sprinkler concept and a standard concept is analyzed.

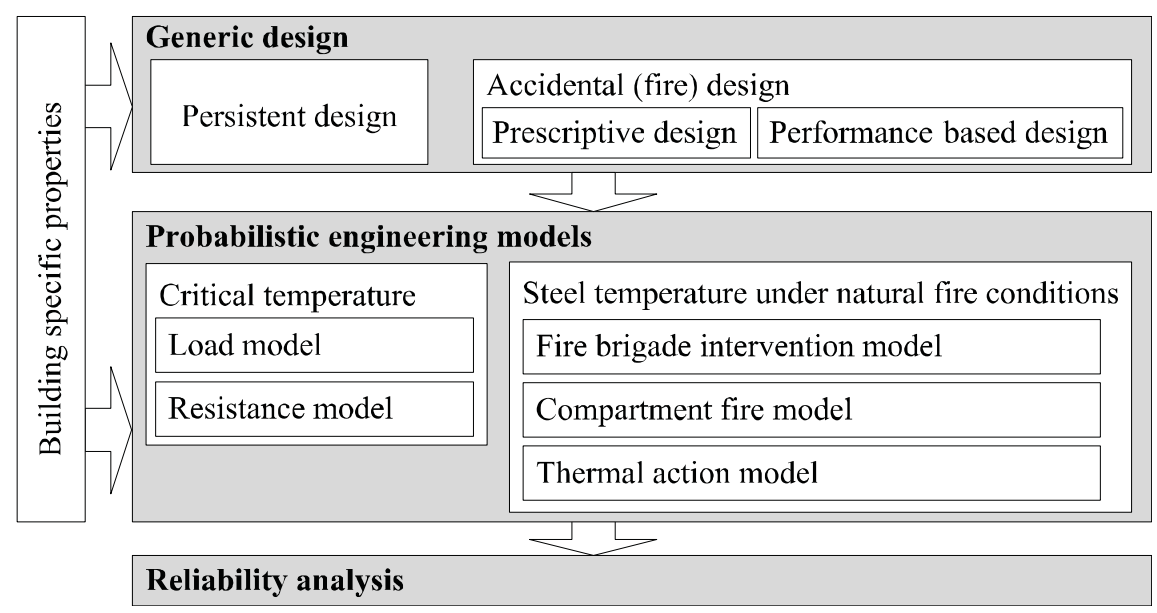

Fig. 1. Assessment of the level of safety.

\section{THE LEVEL OF SAFETY IN STRUCTURAL FIRE DESIGN}

The level of safety in fire safety engineering is achieved through implementing active and passive fire safety measures in the design of the building. Depending on national codes two different methods are used to implement those measures: a performance based design or a prescriptive design.

\section{Prescriptive design}

The level of safety of the prescriptive design is achieved by implementing structural requirements which are explicitly set by the fire authorities. These requirements differ depending on the risk classification of the building classes, i.e. considering the probability of a fire event and its consequences. Therefore, the prescriptive design is able to consider buildings properties only in a limited way, e.g. by differentiation in the occupancy, amount of stories, etc. Usually, the regulations provide a fire resistance class for the load 
bearing capacity $\mathrm{R}$ of a structural member, e.g. R30, R60, R90, etc. This resistance class includes a time limit corresponding to the time where the load bearing capacity of a structural member maintains its function under a standard fire exposure (ISO-834). This time limit is related to the risk classification of the building and is generally provided by the fire authorities.

\section{Performance based design}

In the performance based design the level of safety for a structure is achieved by setting a performance criterion for a limit state. The design has to be adapted to fulfill this criterion. Following JCSS [2] and EN 1990 [3] such a criterion - not only for the fire design - is given by the target reliability index $\beta_{t}$ for the ultimate limit state. This target reliability index is linked to the annual tolerable probability of failure $P_{f, t}$ of a structure. Accordingly, the design has to be chosen in a way that the probability of failure of a structure $P_{f}$ does not exceed the tolerable probability of failure $P_{f, t}$. This probability of failure $P_{f}$ can be assessed through a probabilistic approach. However, this approach requires a lot of experience in uncertainty quantification, reliability analysis and numerical computation and is not suitable for engineering practice.

The semi-probabilistic design is a simplification of the probabilistic approach and builds the basis for different codes like the Eurocode. The principle of this design is to multiply the characteristic values of the basis variables with a factor. Those factors are calibrated towards the target reliability index $\beta_{t}$ (Faber \& Sørensen [8]). Hence, designing a structure with the semi-probabilistic approach - in the ideal case - the failure probability of the structure $P_{f}$ corresponds to the tolerable probability of failure $P_{f, t}$.

Such a calibration has been done by the European research project "Natural Fire Safety Concept" by Schleich et al. [1] where reduction factors for the characteristic fire load have been calibrated. Those reduction factors have been integrated in the EN 1991-1-2 Annex E [5] and consider the occupancy type, the floor area of the building and active fire safety measures, i.e. sprinkler, automatic fire detection devices, etc.

\section{Quantification of the level of safety and performance of a design}

For both, the prescriptive and the performance based design, a common quantification of the level of safety is provided through the reliability assessment of a structure. According to JCSS [2] the reliability of a structure can be expressed through the generalized reliability index $\beta$ and is defined as $\beta=-\Phi^{-1}\left(P_{f}\right)$ where $P_{f}$ is the annual probability of failure and $\Phi^{-1}($.$) is the inverse of the cumulative standard normal distribution.$ Methods to assess structural reliability are discussed e.g. in Hasofer et al. [9] and in Melchers [10]. A common quantification of the level of safety allows, on one hand, to compare the level of safety of the prescriptive and the performance based design. On the other hand, the reliability index $\beta$ can be compared with a target reliability index $\beta_{t}$. According to EN 1990 [3] a target reliability index of $\beta_{t}=4.7$ is proposed for medium consequences. This corresponds to a target annual probability of failure of $P_{f, t} \approx 10^{-6}$ of the structure. The performance of a design format can be assessed by comparing the target annual failure probability $P_{f, t}$ with the reliability of the structure under realistic fire conditions for different buildings $P_{f}$. Thus, the level of safety should be the same for all types of buildings. A design which deviates from the target reliability might not be efficient regarding allocation of resources related to economic purposes as well as to life safety, e.g. providing too high costs for safety measures $\left(P_{f}<P_{f, t}\right)$ or providing not acceptable human or financial losses $\left(P_{f}>P_{f, t}\right)$.

\section{GENERIC DESIGN}

Based on different design situations the requirements for structural resistance can be assessed. The design situation includes e.g. persistent design situation (normal use), accidental design situation (exceptional conditions, e.g. fire), seismic and transient design situation. In this paper only the persistent and accidental design situation (fire) are considered. The generic design is conducted for a protected steel member of a structure.

\section{Generic representation of the persistent design situation}

The persistent design situation determines the mechanical resistance $\mathrm{R}_{d}$ to be provided to resist a certain design load $\mathrm{E}_{d}$. The most general form to formulate the requirement for mechanical resistance of a steel member is given through the ultimate limit state $g_{d}$ in Eq. 1. For the generic design of a structure the 
amount of the mechanical resistance is considered through a design variable $z$. In this variable all structural and mechanical parameters (beam length, moment of inertia, etc.) are implicitly considered. The optimal design variable $z_{\text {opt }}$ is achieved by setting $g_{d}=0$ and depends on the design load $\mathrm{E}_{d}$, the characteristic value of the limiting steel strength $r_{a, k}$ (e.g. yield stress) and the partial safety factor for the resistance $\gamma_{M}$.

$$
g_{d}=\mathrm{R}_{d}-\mathrm{E}_{d}=z \cdot \frac{r_{a, k}}{\gamma_{M}}-\mathrm{E}_{d} \geq 0 \quad \Rightarrow \quad z_{o p t}=\frac{\gamma_{M} \mathrm{E}_{d}}{r_{a, k}}
$$

\section{Generic representation of the accidental design situation for the fire exposure}

The degree of utilization $\mu_{0}$ can be used for the design at elevated temperatures. This degree represents the amount of the required mechanical resistance to sustain a fire. The degree of utilization can only be used for the design when the load bearing capacity is directly proportional to the effective yield strength [11] i.e. for tension members where different types of non-linearities (like buckling) can be excluded. The analyses in this paper respect this limitation. According to Ellingwood [12] the probability of a coincidence of a fire with maximum live load, miscellaneous roof live load, significant wind loads or earthquakes is negligible for fire design (but not conditional actions like fire after earthquake). Therefore, only the permanent load (dead load $d_{k}$ ) and the frequent value of the life load $l$ according to EN 1990 [3] $\left(\psi_{1} l_{k}\right)$ are considered in the generic design. By introducing a load ratio $\alpha=l_{k} /\left(l_{k}+d_{k}\right)$ the degree of utilization can be expressed just through partial safety factors $\left(\gamma_{i}\right.$ and $\left.\psi_{i}\right)$ and the load ratio $\alpha$ :

$$
\mu_{0}=\frac{\mathrm{E}_{f i, d}}{\mathrm{R}_{f, d, 0}}=\frac{\mathrm{E}_{f i, d}}{z_{o p t} \cdot r_{a, k} / \gamma_{M, f i}}=\frac{\frac{1}{\alpha} \cdot(1-\alpha)+\psi_{1}}{\frac{\gamma_{M}}{\gamma_{M f i}} \cdot\left(\gamma_{G} \cdot \frac{1}{\alpha} \cdot(1-\alpha)+\gamma_{Q}\right)}
$$

The reduction of the yield strength on elevated temperatures can be described as a function of the steel temperature $\theta$ and is given e.g. in EN 1993-1-2 [13]. Based on this dependency the critical steel temperature $\theta_{\text {crit }}$ is derived, e.g. the temperature where a structural collapse occurs. The limit state on the temperature domain is formulated through:

$$
g_{d, f i}=\theta_{c r i t}-\theta_{d}=\underbrace{39.19 \ln \left[\frac{1}{0.9674 \mu_{0}^{3.833}}-1\right]+482}_{\theta_{\text {crit }}}-\theta_{d} \geq 0
$$

The thermal resistance of a steel structure is chosen such that the design value of the steel temperature $\theta_{d}$ does not exceed the critical temperature $\theta_{\text {crit }}$. The thermal resistance of a protected steel member is represented through the massivity factor $z_{f}$ :

$$
z_{f i}=\frac{A_{p}}{V} \cdot \frac{\lambda_{p}}{d_{p}}
$$

The massivity factor $z_{f i}$ is used to assess the thermal resistance of a steel member generically and depends on the section factor $A_{p} / V$ of a steel profile, the thermal conductivity of the protection material $\lambda_{p}$ and the thickness of the protection material $d_{p}$. Based on the massivity factor $z_{f i}$ and the gas temperature $\Theta_{g}(t)$ the steel temperature $\theta(t)$ is assessed through the simplified design method described in EN 1993-1-2 [13] as illustrated in Fig. 2. Depending whether a performance based or a prescriptive design is used the fire exposure defers.

For the prescriptive design the design value of the steel temperature $\theta_{d, \mathrm{R}}$ is derived from the standard fire curve (ISO curve) $\Theta_{g, \mathrm{ISO}}(t)$ and from the fire resistance criterion for the load bearing capacity R (Fig. 2a). The fire resistance criterion defines the duration, e.g. $\mathrm{R}=30 \mathrm{~min}, \mathrm{R}=60 \mathrm{~min}$, etc., where the load bearing 
capacity of a steel member under standard fire exposure is maintained. The design value for the steel temperature $\theta_{d, \mathrm{R}}$ is derived from:

$\theta_{d, \mathrm{R}}\left(z_{f i}, \mathbf{x}_{\mathbf{d}}, \mathbf{y}\right)=\theta\left(\mathrm{R} \mid \Theta_{g, \mathrm{ISO}}(t), z_{f i}\right)$

For the performance based design the parametric fire exposure according to EN 1991-1-2 [5] is used. The parametric fire exposure describes the gas temperature per time $\Theta_{g, \mathrm{EN}}\left(t \mid \mathbf{x}_{\mathbf{d}}, \mathbf{y}\right)$ and depends on fire (or event) specific design values $\mathbf{x}_{\mathbf{d}}=\left\{q_{d}, t_{\alpha, d}\right\}$ (e.g. design value of the fire load $q_{d}$ and design value of the fire growth rate $t_{\alpha, d}$ ) and building (object) specific properties $\mathbf{y}=\left\{a_{f}, o, b\right\}$ (e.g. the room geometry $a_{f}$, the ventilation conditions $o$ and the thermal properties of the boundary of enclosure $b$ ). EN 1991-1-2 [5] considers, among other, active fire protection measures by calibrated reduction factors for the characteristic fire load [1]. The design value of the steel temperature $\theta_{d, \mathrm{EN}}$ is derived from the maximal steel temperature that is reached under parametric fire exposure:

$\theta_{d, \mathrm{EN}}\left(z_{f i}, \mathbf{x}_{\mathbf{d}}, \mathbf{y}\right)=\max \left\{\theta\left(t \mid \Theta_{g, \mathrm{EN}}\left(t \mid \mathbf{x}_{\mathbf{d}}, \mathbf{y}\right), z_{f i}\right)\right\}$

For an optimal accidental design, the massivity factor $z_{f, \text { opt }}$ is chosen such that $g_{d, f i}=0$, i.e. that the design value of the steel temperature $\theta_{d}\left(z_{f i, o p t}\right)$ is equal to the critical steel temperature $\theta_{\text {crit }}$ (Fig. 2a). This optimization problem is solved numerically by minimizing the error term $\left|\theta_{d}\left(z_{f i}\right)-\theta_{\text {crit }}\right|$. The structural design is now characterized generically by two design variables: one for mechanical resistance $z_{\text {opt }}$ and one for thermal resistance $z_{f i, o p t}$. In the case of performance based design the optimal design depends on fire (or event) specific design values $\mathbf{x}_{\mathbf{d}}$ and on building specific properties $\mathbf{y}$.

a)

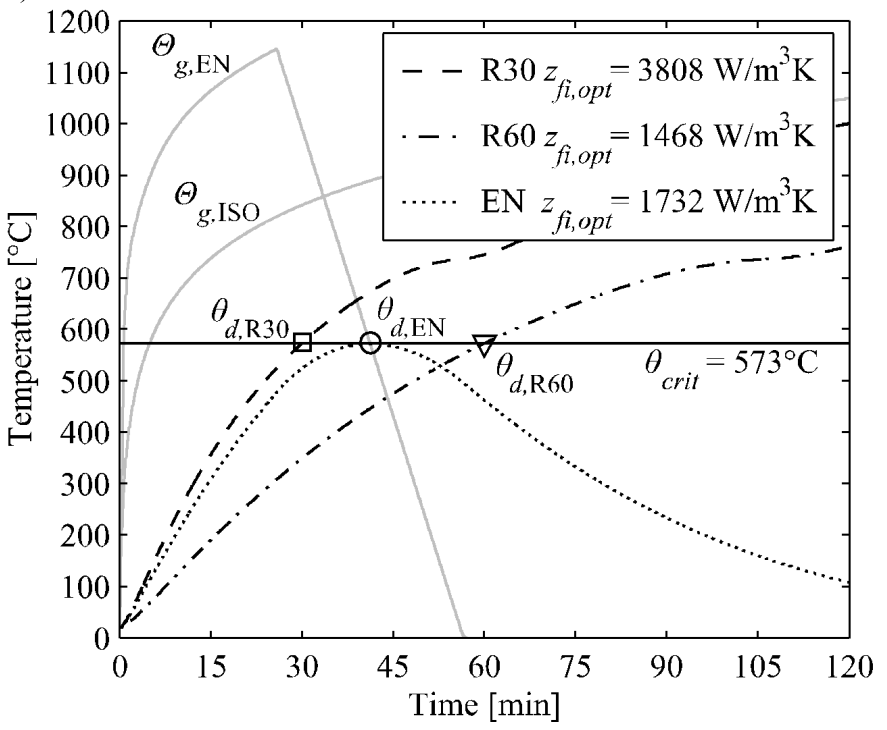

b)

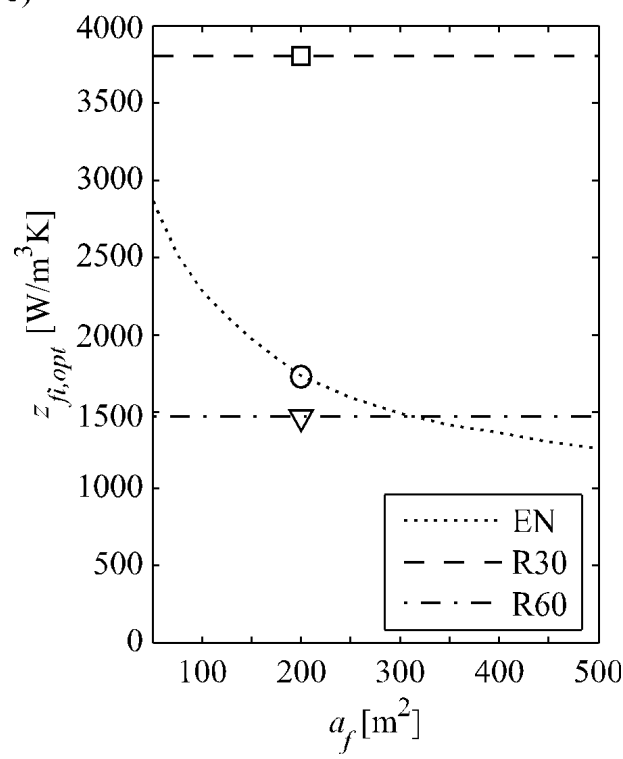

Fig. 2. a) Generic optimal design for $o=0.1 \mathrm{~m}^{1 / 2}$ and $a_{f}=200 \mathrm{~m}^{2}$;

b) Required thermal resistance for $o=0.1 \mathrm{~m}^{1 / 2}$.

\section{Representation of the building}

The building is represented through building specific properties $\mathbf{y}$. In this study five building specific properties and three induced building specific properties are used (Table 1). The induced building specific properties are derived from the five building specific properties assuming a quadratic floor area of the compartment. In this paper, the floor area $a_{f}$ and the opening factor $o$ are varied to analyze their effect on the level of safety. The other building specific properties are set to be constant (Table 1). The effect of the floor area on the optimal thermal resistance $z_{f, \text { opt }}$ is illustrated in Fig. $2 \mathrm{~b}$ according to a prescriptive design 
(R30 and R60) and a performance based design (EN). In Fig. 2b a qualitative estimation of the level of safety of the corresponding design can already be made. A higher thermal resistance of a structure will lead to a higher level of safety. Thus, for building properties $a_{f}<300 \mathrm{~m}^{2}$ and $o=0.1 \mathrm{~m}^{1 / 2}$ the level of safety of a prescriptive design (R60) is higher compared to a performance based design (EN).

Table 1. Generic representation of the building (retail) by building properties $\mathbf{y}$.

\begin{tabular}{llll}
\hline \multicolumn{2}{l}{ Building specific properties } & \multicolumn{2}{l}{ Induced building specific properties } \\
\hline$a_{f}$ & Floor area of the compartment $\left[\mathrm{m}^{2}\right]$ & $h_{e q}=0.8 h$ & Ventilation height $[\mathrm{m}]$ \\
$o$ & Opening factor $\left[\mathrm{m}^{1 / 2}\right]$ & $a_{t}=4 h\left(a_{f}\right)^{1 / 2}+2 a_{f}$ & Total area of enclosure $\left[\mathrm{m}^{2}\right]$ \\
$b=1000$ & Thermal absorptivity of the & $a_{V}=o \cdot a_{t} /\left(h_{e q}\right)^{1 / 2}$ & Total area of vertical openings \\
& enclosure $\left[\mathrm{J} / \mathrm{m}^{2} \mathrm{~K} \mathrm{sec}\right.$ & \\
$\alpha=0.5$ & Load ratio $[-]$ & & \\
$h=3.5$ & Compartment height $[\mathrm{m}]$ & & \\
\hline
\end{tabular}

\section{PROBABILISTIC ENGINEERING MODELS}

The structural design is analyzed under realistic fire conditions to quantify the level of safety. Accordingly, the physical processes are represented by engineering models. It is important to consider all the relevant processes that affect the structure including the fire exposure, the fire brigade intervention, the thermal response of the structure, the load conditions and the mechanical resistance. In this chapter the engineering models are discussed representing the physical processes. Under real conditions the structure is exposed to random effects due to natural variability. Therefore, a probabilistic approach is used to model those effects. Statistical data are used to quantify those uncertainties and can be represented by probabilistic models. Where no statistical data are available the probabilistic models can be estimated through engineering judgment. The probabilistic models that are used in this paper are listed in Table 2.

\section{Limit state function}

The limit state function for structural failure of a steel member in Eq. 7 is formulated analogously to Eq. 3 in the temperature domain. The difference to Eq. 3 is that the fire specific variables $\mathbf{X}$ are introduced as random variables where the building specific properties $\mathbf{y}$ are chosen to be deterministic. Based on those deterministic variables a parameter study is made to assess the effect of the building specific indicators on the reliability of a structure.

Table 2. Probabilistic models for fire specific random variables $\mathbf{X}$ for Swiss retail buildings.

\begin{tabular}{|c|c|c|c|c|c|c|}
\hline Variables & $X$ & $x$ & Distribution & $\mathrm{E}[X]$ & $\operatorname{CoV}[X]$ & Reference \\
\hline Fire load $\left[\mathrm{MJ} / \mathrm{m}^{2}\right]$ & $Q$ & $q$ & Lognormal & 600 & 0.30 & [5] \\
\hline Heat release rate $\left[\mathrm{kW} / \mathrm{m}^{2}\right]$ & $R H R_{f}$ & $r h r_{f}$ & Normal & 200 & 0.20 & [4], estimated \\
\hline Fire growth time [sec] & $T_{\alpha}$ & $t_{\alpha}$ & TruncLN $^{\text {a) }}$ & 361 & 0.55 & {$[15,16]$} \\
\hline Max. treatable fire size $\left[\mathrm{m}^{2}\right]$ & $A_{\text {Limit }}$ & $a_{\text {Limit }}$ & Normal & 200 & 0.15 & [4], estimated \\
\hline Detection time [min] & $T_{\text {Detect }}$ & $t_{\text {Detect }}$ & TruncGam ${ }^{\text {b) }}$ & 3.67 & 1.11 & {$[15,16]$} \\
\hline Call time [min] & $T_{\text {Call }}$ & $t_{\text {Call }}$ & Lognormal & 2.50 & 0.75 & [15] \\
\hline Dispatch time $[\mathrm{sec}]$ & $T_{\text {Disp }}$ & $t_{\text {Disp }}$ & Lognormal & 155 & 0.20 & [17], estimated \\
\hline Turnout and travel time [min] & $T_{\text {TOut }+T}$ & $t_{T O u t+T}$ & Lognormal & 6.60 & 0.52 & {$[17,18]$} \\
\hline Setup time ${ }^{\mathrm{c})}[\mathrm{min}]$ & $T_{\text {Setup }}$ & $t_{\text {Setup }}$ & Lognormal & 3.50 & 0.15 & [19], estimated \\
\hline Dead load $\left[\mathrm{kN} / \mathrm{m}^{2}\right]$ & $D$ & $d$ & Normal & $4.28^{\mathrm{d})}$ & 0.10 & [2] \\
\hline Sustained live load $\left[\mathrm{kN} / \mathrm{m}^{2}\right]$ & $L_{S}$ & $l_{s}$ & Gamma & 0.90 & 1.34 & [2] \\
\hline Limiting steel strength $\left[\mathrm{N} / \mathrm{mm}^{2}\right]$ & $R_{a}$ & $r_{a}$ & Lognormal & 264 & 0.07 & [2] \\
\hline
\end{tabular}

a) Truncated Lognormal distribution (at $800 \mathrm{sec}$ ) to exclude smoldering fires

b) Truncated Gamma distribution (at $15 \mathrm{~min}$ ) to exclude large detection times due to smoldering fires

c) Depends on building properties [19] (single-floor building and good accessibility assumed)

d) Derived from $\alpha=l_{k} /\left(l_{k}+d_{k}\right)$ and [2]

The critical steel temperature $\theta_{\text {crit }}$ in Eq. 3 is assessed based on a conservative design formula. For simplicity this formula is used in the reliability analysis neglecting the variability of the steel strength at high temperatures. This results in a (safe) bias in the prediction of the level of safety. 
$G_{f i}(\mathrm{X}, \mathrm{y})=\underbrace{39.19 \ln \left[\frac{1}{0.9674 \mu_{0}\left(\mathrm{X}, z_{\text {opt }}\right)^{3.833}}-1\right]+482}_{\text {Critical steel temperature } \theta_{\text {crit }}}-\underbrace{\max \left\{\theta\left(t \mid \Theta \Theta_{g}(t \mid \mathrm{X}, \mathrm{y}), z_{f i, \text { opt }}\right)\right\}}_{\begin{array}{c}\text { Maximal steel temperature under } \\ \text { natural fire conditions }\end{array}}$

\section{Compartment fire model}

Instead of using the parametric fire curves given in EN 1991-1-2 [5] the zone model OZone [14] is used to assess the gas temperature $\Theta_{g}(t \mid \mathbf{X}, \mathbf{y})$ in the compartment depending on fire specific random variables $\mathbf{X}$ and on building specific properties $\mathbf{y}$. Using a more advanced fire model as OZone allows to check how well the parametric fire curves represents realistic fires. Further, it is possible to consider active fire safety measures based on measurable quantities.

A fire can be characterized through three phases: the pre-flashover phase, the fully engulfed fire (postflashover) and the decay phase (Fig. 3). The pre-flashover phase of a fire is usually modeled by a $t^{2}$ approach characterizing the increase of the rate of heat release (see Fig. 3). The fire spread is assumed to be constant and spreading radial in all directions. The increase of the heat release is described through the fire growth parameter $t_{\alpha}$ and is defined as the time needed for the fire to reach a rate of heat release of $1000 \mathrm{~kW}$. The fire spread area $a_{S}$ can be assessed through:

$$
a_{S}(t)= \begin{cases}1000 \mathrm{~kW}\left(\frac{t}{t_{\alpha}}\right)^{2} \frac{1}{r h r_{f}} & \text { pre-flashover phase } \\ a_{f} & \text { full engulfed fire }\end{cases}
$$

The full engulfed fire - when the fire engulfs the total compartment area $a_{f}$ - is characterized through a constant rate of heat release depending on the fire regime, limited by the fuel or the oxygen. A fuel controlled fire reaches the maximal rate of heat release $r h r_{f}$ and depends on the surface and the material properties of the combustible materials. For ventilation controlled fires the maximal rate of heat release is limited by the available oxygen. For the thermal analysis with OZone only the fuel controlled rate of heat release is needed. The program recognizes the limited availability of oxygen and adapts the rate of heat release for ventilation controlled fires [14]. The fire load $q$ determines the full engulfed fire. According to EN 1991-1-2 [5] the linear decay phase starts after 70\% of the total fire load $q \cdot a_{f}$ has been combusted.

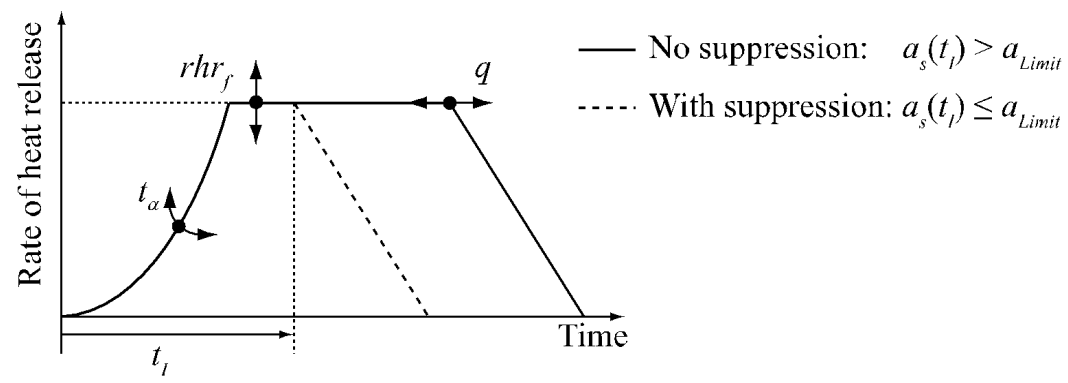

Fig. 3. Qualitative illustration of the rate of heat release.

\section{Fire brigade intervention model}

The success of the fire brigade depends on the size of the fire brigade and their equipment and on the intervention time $t_{I}$ when the fire brigade starts with their fire suppression activities. This time $t_{I}$ consist on several consecutive time intervals as illustrated in Fig. 4 and can be expressed through the sum of all these times (Eq. 9). These time intervals are dependent on the performance level of the local fire brigade (dispatch, turnout, travel and setup time) and on building specific properties (setup time) [19]. The probabilistic models describing those variables are discussed in De Sanctis et al. [16] and are listed in Table 2. Probabilistic models to consider technical fire safety measures that reduce especially the detection and the call time (e.g. detection systems) are discussed in De Sanctis et al. as well. 


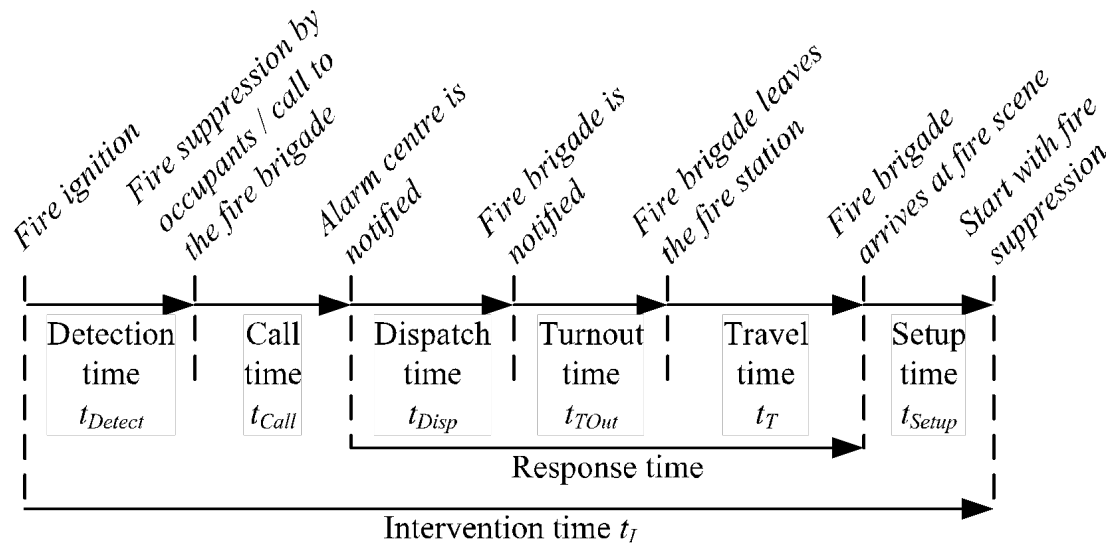

Fig. 4. Composition of the intervention time of the fire brigade.

If the fire is grown too large, the suppression of the fire might not be possible and a full burnout will occur. The fire brigade must then focus on preventing further fire spread. Hosser et al. [4] proposed a model based on a maximal treatable fire size $a_{\text {Limit }}$. This fire size is used as an indicator for the fire suppression capability of the fire brigade and includes implicit the crew size of the fire brigade, the extinguishing equipment, accessibility of the fire, etc. If the fire grows larger than this area $a_{S}\left(t_{I}\right)>a_{\text {Limit }}$ then a complete burnout must be accepted; otherwise the fire suppression action starts reducing the rate of heat release. It is assumed that the decay phase starts at the intervention time $t_{I}$ of the fire brigade (Fig. 3). The relation $a_{S}\left(t_{I}\right)>a_{\text {Limit }}$ determines the probability of failure of the fire suppression by the fire brigade.

\section{Thermal action on a steel member}

The maximal steel temperature is assessed based on the gas temperature $\Theta_{g}(t)$ derived from the compartment fire model. The simplified calculation method described in EN 1993-1-2 is used to assess the steel temperature $\theta\left(t \mid \Theta_{g}(t \mid \mathbf{X}, \mathbf{y}), z_{f i}\right)$. According to a global sensitivity analysis carried out by De Sanctis et al. [16], the uncertainty associated with the fire growth time has a major influence on the maximal steel temperature if the fire brigade has been modeled as presented in this paper. The analysis indicates a high non-linearity regarding the interaction effects between the random variables; especially due to the interaction of the fire brigade intervention and the fire spread. This is in contrast to the case, when the fire brigade intervention is independently modeled as done in Schleich et al. [1]; e.g. assuming an independent probability of failure for the fire brigade intervention. Then the model behaves almost linear and the most sensitive parameter is the fire load.

\section{Load and resistance model}

As a fire is a rare event, a structure is likely to be loaded only to a part of the design load when the fire occurs. For the reliability analysis the - so called - point in time distribution of the load during a fire event is used. Ellingwood [12] concluded that the major participation of the load in a fire is due the dead load $d$ and the sustained component of the live load $l_{S}$. The degree of utilization is assessed considering the random variable of the load, the limiting steel strength $r_{a}$ and the optimal design variable $z_{\text {opt }}$ for the persistent design situation. The probabilistic models of the variables are modeled according to JCSS [2] and are listed in Table 2.

$$
\mu_{0}\left(\mathbf{x}, z_{\text {opt }}\right)=\frac{d+l_{S}}{z_{\text {opt }} \cdot r_{a}}
$$




\section{RELIABILITY ANALYSIS}

The reliability of a structure is measured through the annual probability of failure $P_{f}$. This probability is assessed based on conditional probabilities of events. In the present case the annual probability of failure of a structure may be assessed through the probability of a fire ignition $P(I)$, the conditional probability of a fire which affects the structure $P(S F \mid I)$ (excluding smoldering fires) and the conditional probability of a structural failure $P(S \mid S F, I)$ :

$$
P_{f}=P(I) \cdot P(S F \mid I) \cdot P(S \mid S F, I)
$$

\section{Probability of fire ignition and conditional probability of a fire which affects the structure}

The probability of fire ignition $P(I)$ can be described through the annual probability of fire occurrence in a building. Fischer et al. [20] provides an empirical model (Eq. 12), which has been developed based on Swiss insurance data, to assess the probability of fire ignition depending on its volume. Here, the volume is expressed through the floor area $a_{f}$ and the compartment height $h$.

$$
P(I)=1.26 \cdot 10^{-4} \cdot\left(\frac{a_{f} \cdot h}{1 \mathrm{~m}^{3}}\right)^{0.44}
$$

Fire ignition in this context is the annual probability of occurrence of a financial loss covered by the building fire insurance company providing the data. Hence, also small fires which are extinguished in an early stage or smoldering fires are included in this probability. To assess the probability of a fire that affects the structure $P(S F \mid I)$ the probability of a suppression by occupants are included, e.g. as $P_{O c c}=0.5$. Further the probability of smoldering fires, e.g. as $P_{\text {Smoldering }}=0.71$, which is derived from the non-truncated distribution of the fire growth time, are included as well.

Further, also the installation of a sprinkler can be considered. It is assumed that an activation of the sprinkler will lead to a fire which does not affect the structure. Thus, the sprinkler can be considered in the reliability analysis through reducing the conditional probability $P(S F \mid I)$ by the failure probability of the sprinkler, e.g. as $P_{\text {Sprinkler }}=0.02$.

\section{Conditional probability of structural failure}

Structural failure can be defined when the limit state function defined in Eq. 7 turns negative. Then the maximal steel temperature exceeds the critical temperature and the structure fails. The conditional probability of a structural failure $P(S \mid S F, I)$ is assessed through the probability of a negative outcome of the limit state:

$$
P(S \mid S F, I)=P\left(G_{f i}(\mathbf{X}, \mathbf{y}) \leq 0 \mid S F, I\right)=\int_{g_{f i}(\mathbf{x}, \mathbf{y}) \leq 0} f_{\mathbf{X}}(\mathbf{x}) \mathrm{d} \mathbf{x}
$$

The probability $P\left(G_{f i}(\mathbf{X}, \mathbf{y}) \leq 0 \mid S F, I\right)$ is in general assessed through a numerical multidimensional integration over the probability densities $f_{\mathbf{X}}($.$) of the random variables \mathbf{X}$ where the limit state function is negative, e.g. $G_{f i}(\mathbf{X}, \mathbf{y}) \leq 0$. Because this numerical integration is computational very expensive, two different methods can be used to assess the probability of failure: simulation methods and approximation methods, e.g. FORM, SORM and surrogate models. Because approximation methods tend to be sensitive to non-linear behavior of the limit state function, in this paper a simulation method named subset simulation [6] is used to estimate the failure probability $P\left(G_{f i}(\mathbf{X}, \mathbf{y}) \leq 0 \mid S F, I\right)$. The basic idea is to express the small failure probability $P(S \mid S F, I)$ as a product of larger conditional failure probabilities $P\left(F_{i+1} \mid F_{i}\right)$ by introducing intermediate failure events (subsets) $F_{i}$ :

$$
P(S \mid S F, I)=P\left(\sum_{i=1}^{m} F_{i}\right)=P\left(F_{1}\right) \prod_{i=1}^{m} P\left(F_{i+1} \mid F_{i}\right) \quad \text { and } \quad F_{i}=\left\{G_{f i}(\mathbf{X}, \mathbf{y}) \leq \Delta_{i}\right\}
$$


The intermediate conditional failure events (subsets) are related to a threshold value $\Delta_{1}>\Delta_{2}>\ldots>\Delta_{m}=0$. The first threshold $\Delta_{1}$ is derived from a crude Monte Carlo Simulation in which the probability of the first subset is equal to $P\left(F_{1}\right) \approx 0.1 \div 0.2$. For the further conditional subsets $i$ the threshold values $\Delta_{i}$ are assessed similar but through a Markov Chain Monte Carlo simulation based on a modified Metropolis-Hasting algorithm introduced by $\mathrm{Au} \&$ Beck [6]. This algorithm generates new realizations of the intermittent failure event $F_{i+1}$ conditional on the realizations of the failure event $F_{i}$. Assessing the individual probability of the conditional intermediate failure event $P\left(F_{i+1} \mid F_{i}\right)$ is computational much more efficient compared to the case where a small failure probability, e.g. $P(\mathrm{~S} \mid \mathrm{SF}, \mathrm{I})$, has to be estimated directly through a Monte Carlo simulation. The subset simulation method is described in Au \& Beck [6] in details. The subset simulation method is implemented in the Matlab ${ }^{\circledR}$ toolbox FERUM [21] which is used to assess the probability. The number of simulations depends on the probability of the conditional intermediate failure events $P\left(F_{i+1} \mid F_{i}\right) \approx 0.1 \div 0.2$ and the intended coefficient of variation $(\mathrm{CoV}<10 \%)$. This $\mathrm{CoV}$ is a measure for the error that occurs by estimating the probability. For larger probabilities (e.g. $>10^{-6}$ ) a few thousand of simulation has been sufficient and for lower probabilities a few ten-thousands of simulation has been required. For small probabilities (e.g. $\left.<10^{-6}\right)$ a higher coefficient of variation $(\mathrm{CoV}>10 \%)$ has been accepted to keep the computational effort in a reasonable range. By comparing the number of simulations $N$ required for a small failure probabilities $P=10^{-k}$ the advantage of the subset simulation, e.g. $N \sim k \cdot 10^{4}$, compared to the crude Monte Carlo simulation, e.g. $N \sim 10^{k+2}$, becomes evident.

\section{THE SAFETY LEVEL OF STRUCTURAL DESIGN UNDER REALISTIC FIRE CONDITIONS}

Based on the reliability analysis the level of safety of a structure that is designed according to a certain code format is assessed. Thus, a comparison of the prescriptive (R30, R60) and performance based (EN) designs is made. The floor area $a_{f}$ and the opening factor $o$ are used to analyze the effect of different buildings properties. The other building specific properties are chosen according to Table 1. Fig. 5-8 in this chapter have all the same format: on the x-axis the floor area is plotted and on the y-axis (logarithmic scale) the annual failure probability $P_{f}$ assessed through Eq. 11 is plotted. The zigzagged shape of some lines can be explained either by the error (especially for very low $P_{f}$ ) made by the estimation of the failure probability (Eq. 14) or by non-linear resp. non-continuous effects of the model.

\section{Bias in the prediction of the failure probability}

It should be noted that due to assumption and simplification used in the probabilistic engineering models the prediction of the failure probability is associated with a certain bias. Thus, to be exact, an absolute statement whether a design is safe or unsafe can actually not be done. Hence, only relative comparisons of the designs are possible. However, it can be assumed that the prediction of the failure probability is in the order of magnitude of the unbiased probability of failure. The target reliability index $\beta_{t}$ is indicated in Fig. 6-8 representing an absolute value that divides the acceptable and the non-acceptable range. But any interpretation should be done with care.

\section{Influence of the fire brigade model}

The calibration of the Eurocode by Schleich et al. [1] has been made by using some assumption that defer to the models presented in this paper, e.g. the fire brigade model. Schleich et al. consider the fire brigade through a probability of failure of the suppression activities independent on the building properties and on the fire conditions. Further, the full burnout is assumed through parametric fire curves without the effect of reducing the rate of heat release through the fire brigade as done in this paper. In Fig. 5 (EN Schleich et al.) the results are illustrated by implementing those assumptions in the model. The probability of failure corresponds almost exactly to the target reliability index $\beta_{t}$. This is unsurprisingly, because this model has been used to calibrate the code format used in the generic design towards the target reliability.

The fire brigade model considers the fire brigade intervention on a more physical way. Though, there are some assumptions made by engineering judgment. One of these assumptions is the maximal treatable fire size of the fire brigade $A_{\text {Limit }}$. The probabilistic model for this parameter is estimated by setting a mean value of $\mathrm{E}\left[A_{\text {Limit }}\right]=200 \mathrm{~m}^{2}$. To assess the influence of this assumption this mean value is reduced to $\mathrm{E}\left[A_{\text {Limit }}\right]=100 \mathrm{~m}^{2}$. In this case (Fig. 5), the fire suppression capability decreases and increases the failure probability of the structure especially for compartment areas $a_{f}<200 \mathrm{~m}^{2}$. 


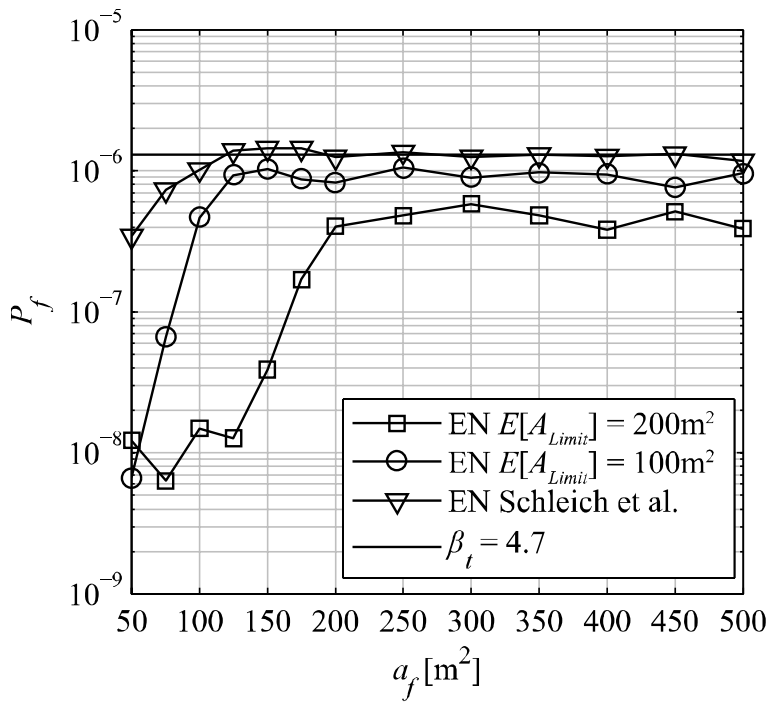

Fig. 5. Influence of the fire brigade intervention for $o=0.10 \mathrm{~m}^{1 / 2}$.

a)

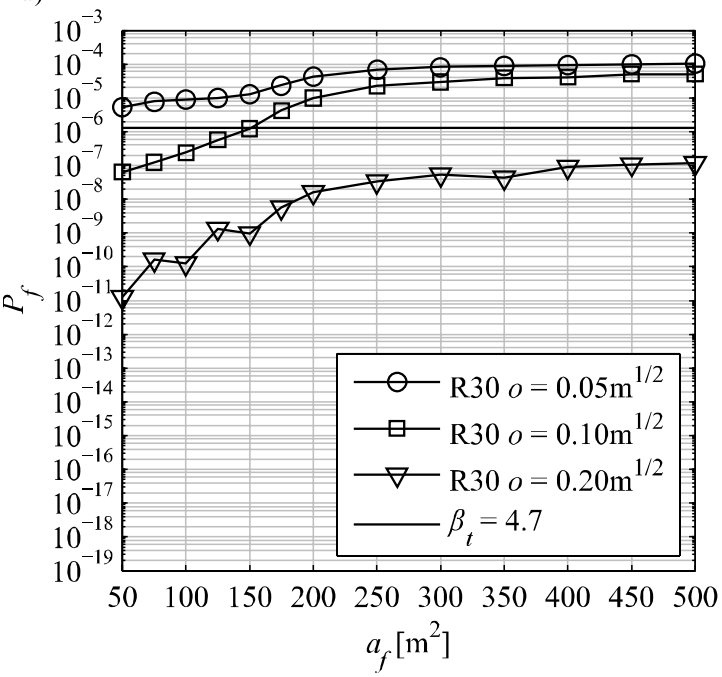

b)

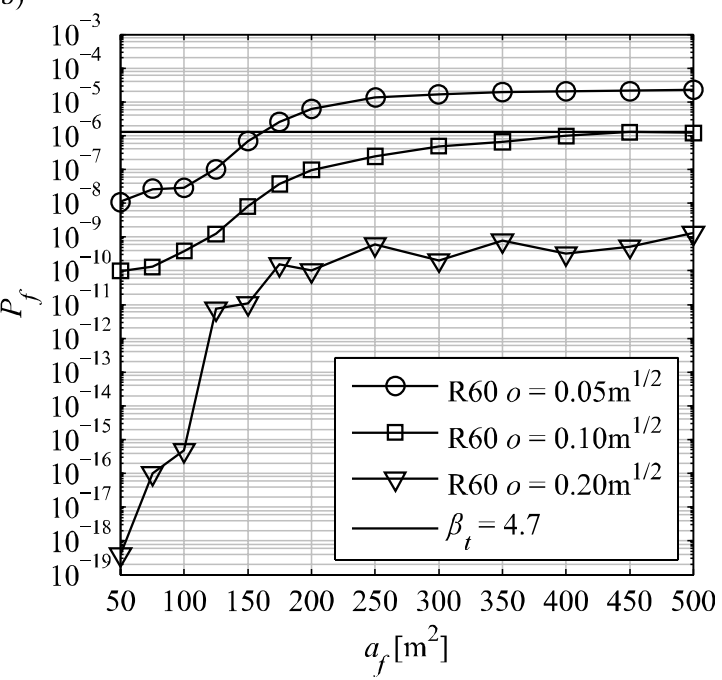

Fig. 6. Level of safety of prescriptive design a) for R30; b) and for R60.

\section{The level of safety of prescriptive design}

The annual failure probability $P_{f}$ of a prescriptive design depends a lot on the building properties (Fig. 6). This is comprehensible because the prescriptive design does not take the building properties (e.g. the floor area) into account (see Fig. $2 b$ ). This leads to a high variation of the annual failure probability $P_{f}$ depending on the building properties.

\section{The level of safety of performance based design}

The lines of the failure probability in Fig. 7 for different opening factors follow almost the same shape. The opening factor $o=0.20 \mathrm{~m}^{1 / 2}$ leads to the lowest failure probabilities. The reason is that large opening factors are physically not so well represented by the parametric fire curves. Further, for floor areas $a_{f}<200 \mathrm{~m}^{2}$ the failure probability is smaller than for higher areas because of two effects: the duration of a fire for small compartments is reduced because of a small amount of fuel and the success of the fire brigade is higher as for larger compartments due to a limited fire spread (see also Fig. 5). The range of the failure probabilities is obviously smaller compared to the prescriptive design. Thus, the performance based design indicates a 
better indifferent treatment of the building specific properties compared with the prescriptive design, especially regarding the opening factors.

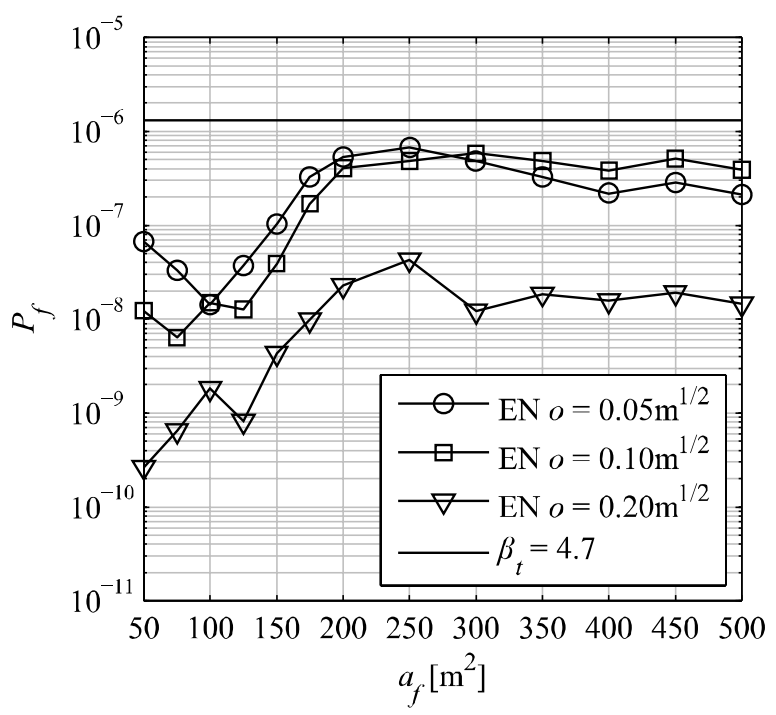

Fig. 7. Level of safety of performance based design.
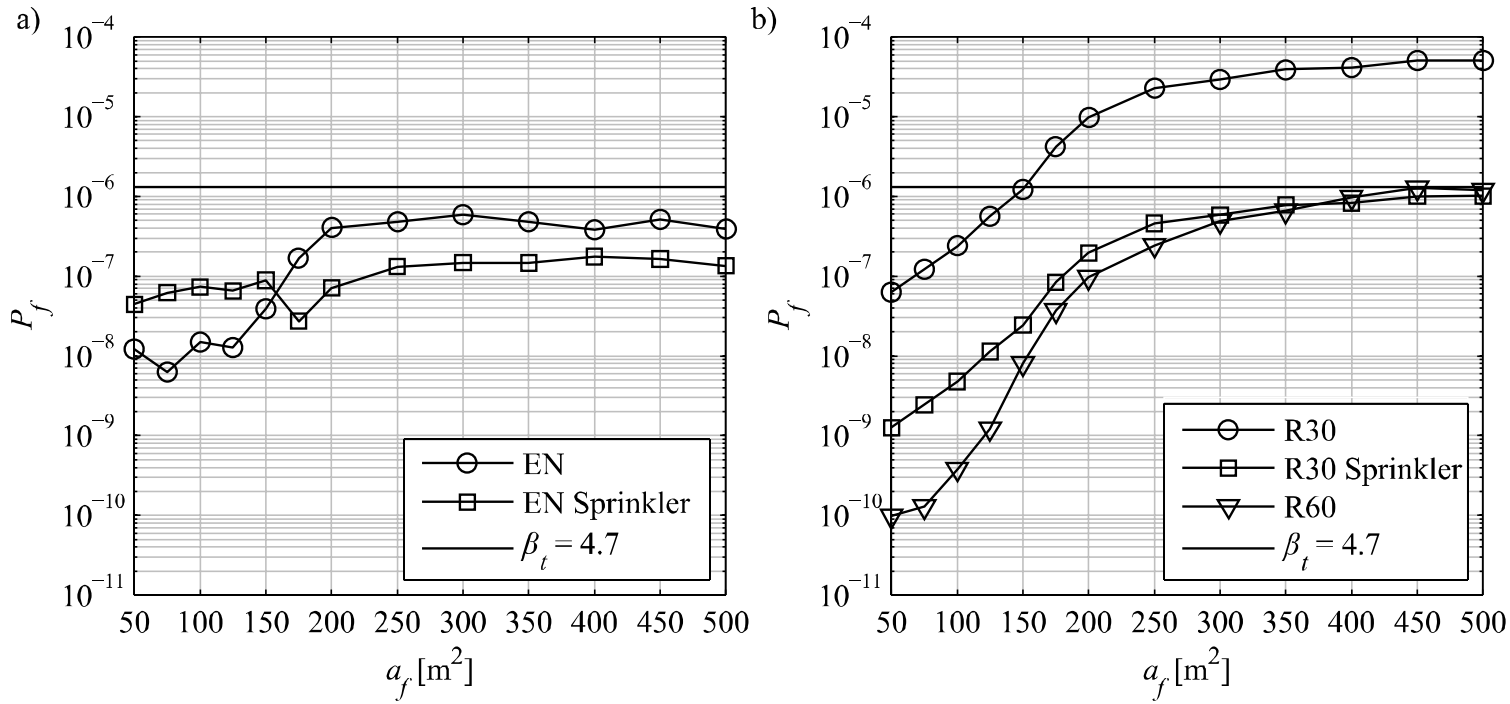

Fig. 8. Equivalence of fire safety measures for a) performance based design; b) prescriptive design $\left(o=0.1 \mathrm{~m}^{1 / 2}\right)$.

\section{Equivalence of fire safety measures}

In EN 1991-1-2 [5] active fire safety measures are considered in the performance based design by reducing the design fire load. Some prescriptive design codes allow to reduce the fire resistance, e.g. from R60 without sprinkler to R30 with sprinkler. The equivalency of the design is verified by comparing the annual failure probability of the standard design $P_{f, \text { Standard }}$ (without an active fire safety measure) and the alternative design $P_{f, \text { Alternative }}$ (with an active fire safety measure). As an example, a sprinkler as an active fire safety measure is considered (Fig. 8).

The prescriptive and the performance based design method indicate a very low probability of failure for small floor areas $\left(a_{f}<150 \mathrm{~m}^{2}\right)$ for the standard design. For those cases, the equivalency is not fully satisfied due to a very low probability of the standard solution. For larger floor areas $\left(a_{f}>150 \mathrm{~m}^{2}\right)$, the equivalency 
of the standard and alternative design is fulfilled or almost fulfilled. Figure 8a indicates even a possible higher reduction of the design fire load for the alternative (performance based) design with sprinkler achieving a better allocation of resources.

\section{CONCLUSION}

A reliability based method is used to quantify the level of safety of prescriptive and performance based design. In this study, the level of safety is quantified through the annual failure probability of the structure. To assess this probability the building and its structural design is represented generically. This facilitates a parametric study of the building specific properties. The generic design assesses the mechanical and thermal requirements for the structure that are required from the codes. The structural design is analyzed under realistic conditions through a probabilistic approach. Within this approach, engineering models are used to represent the physical processes in a fire event including the intervention of the fire brigade and compartment fire modeling by a zone model. To assess the failure probability of the structure, an advanced Monte Carlo Simulation named subset simulation is used. The subset simulation reduces the computational effort for the reliability analysis by many times and made a parametric study possible. The reliability analysis is applied to structural design of retail buildings according to prescriptive and performance based design methods for different building properties.

The level of safety of the prescriptive design is very sensitive to the building specific properties especially concerning the ventilation conditions. Thus, depending on the fire resistance requirement and the building properties, the structures might have high or very low failure probabilities, especially because some building properties are not taken into account. In the performance based design, however, the building properties can be considered and markedly influence the safety level. Hence, applying a prescriptive design might be only suitable for a building portfolio in which building properties varies only in a small range. Otherwise, it might results in non-economical or even in non-safe buildings depending on the defined requirements for the structure that are usually set by the fire authorities.

In the present study the performance based design is applied by using parametric fire curves and using reduction factors according to EN 1991-1-2 Annex E [5]. Advanced probabilistic engineering models are used to check how well the design concept represents the realistic fire conditions. The level of safety of the performance based design varies - within the parameter study - in a smaller range compared to the prescriptive design. Further, the overall tendency of the performance based design provides equal or even lower failure probabilities of the structure compared to the prescriptive design. Exception, are cases where the floor area are small - but in those cases the failure probabilities of the prescriptive design are very low anyway because of the favorable conditions for the fire brigade intervention. There, the design concept can be improved by taking the fire brigade intervention more adequately into account. This will affect structural design requirements especially for small compartments. Further, the design concept can be improved by consider the large ventilation conditions more adequately.

The presented reliability based method is able to prove equivalency between standard structural fire design concepts and alternative design concepts that include structural and technical fire safety measures.

Finally, the introduced methodology combined with the low computational costs for the reliability analysis can be used in the future to calibrate safety factors for the performance based design considering advanced fire models and the fire brigade intervention.

\section{REFERENCES}

[1] Schleich J.-B., Cajot L.-G., Pierre M., Joyeux D., Aurtenetxe G., Unanua J., Competitive steel buildings through natural fire safety concepts, ECSC, 2002.

[2] JCSS, Probabilistic Model Code, The Joint Committee on Structural Safety, 2001.

[3] EN 1990, (Eurocode 0): Basis of structural design, CEN, Brussel, 2002.

[4] Hosser D., Weilert A., Klinzmann C., Schnetgöke R., Albrecht C., Sicherheitskonzept zur Brandschutzbemessung, Institut für Baustoffe, Massivbau und Brandschutz, Braunschweig, 2008.

[5] EN 1991-1-2, (Eurocode 1): Actions on structures - Part 1-2: General actions - Actions on Structures Exposed to Fire, CEN, Brussel, 2002. 
[6] Au S.K., Beck J.L., (2001) Estimation of small failure probabilities in high dimensions by subset simulation, Probabilistic Engineering Mechanics, http://dx.doi.org/10.1016/S02668920(01)00019-4

[7] Au S.K., Wang Z.-H., Lo S.-M., (2007) Compartment fire risk analysis by advanced Monte Carlo simulation, Engineering Structures, http://dx.doi.org/10.1016/j.engstruct.2006.11.024

[8] Faber M.H., Sørensen J.D. "Reliability based code calibration - the JCSS approach", Proceedings to the 9th International Mechanisms for Concrete Structures in Civil Engineering ICASP. San Francisco, USA, 2003.

[9] Hasofer A.M., Beck V.R., Bennetts I.D., Risk Analysis in Building Fire Safety Engineering, Elsevier, Amsterdam, 2007.

[10] Melchers R.E., Structural Reliability Analysis and Prediction (2 $2^{\text {nd }}$ ed), John Wiley \& Sons, 2002.

[11] Franssen J.-M., Vila Real P., Fire Design of Steel Strunctures, Eurocodes Design Manuals, 2010.

[12] Ellingwood B.R., (2005) Load Combination Requirements for Fire-resistant Structural Design. Journal of Fire Protection Engineering, http://dx.doi.org/10.1177/1042391505045582

[13] EN 1993-1-2, (Eurocode 3): Design of steel structures - Part 1-2: General rules - Structural fire design, CEN, Brussel, 2002.

[14] Cadorin J., Pintea D., Franssen J., The Design Fire Tool OZone V2.0 - Theoretical Description and Validation on Experimental Fire Tests, University of Liege, Belgium, 2001.

[15] Holborn P.G., Nolan P.F., Golt J., (2004) An analysis of fire sizes, fire growth rates and times between events using data from fire investigations, Fire Safety Journal, http://dx.doi.org/10.1016/j.firesaf.2004.05.002

[16] De Sanctis G., Kohler J., Fontana M., "On the use of fire brigade statistics for structural fire safety engineering”, Proceedings to Applications of Structural Fire Engineering, Prague, 2013.

[17] FKS Feuerwehr Koordination Schweiz, Feuerwehr 2015, Switzerland, 2009.

[18] London Fire Brigade, Our Performance 2010/11, London, UK, 2011.

[19] Klinzmann C., "Usage of statistical data for a probabilistic model for fire department response", Fire Brigade Reports and Investigations, COST TU0904, 2013.

[20] Fischer K., Kohler J., Fontana M., Faber M.H., Wirtschaftliche Optimierung im vorbeugenden Brandschutz, Institute of Structural Engineering, ETH, Zurich, 2012.

[21] Bourinet J.-M., FERUM 4.1 User's Guide (Finite Element Reliability Using Matlab), Institut Français de Mécanique Avancée, Clermont-Ferrand, France, 2010. 